\title{
Validity of the SCID-II in Diagnosing General Personality Disorder
}

\author{
F. Dereboy ${ }^{1}$, M. Eskin², Ç. Dereboy ${ }^{1}$ \\ ${ }^{1}$ Department of Psychiatry, Adnan Menderes University, Aydın, Turkey. \\ 2 Department of Psychology, Koç University, İstanbul, Turkey.
}

\section{Objectives:}

To investigate the validity of the SCID-II diagnoses and scores against general Personality Disorder (PD) diagnoses assigned by a LEAD (longitudinal, expert, all data) panel (Spitzer, 1983).

\section{Background:}

Despite the recent trend emphasizing the primacy of assessing the severity and presence of general PD rather than a series of specific PD types, research addressing the validity of the SCID-II in diagnosing general PD is scarce.

\section{Materials and Methods:}

The DSM-III-R adjusted SCID-II interviews of 120 volunteering mental health consumers were performed by graduate students in clinical psychology. The LEAD panel consisting of three faculty members (a psychiatrist and two clinical psychologists) evaluated each subject in terms of presence or absence of general PD. We computed the sensitivity and specificity values as sample estimates of diagnostic accuracy for various SCID-II diagnoses and SCID-II total scores with cut-offs suggested by the ROC analysis. Chance corrected agreement between the index and the reference diagnoses were assessed by means of kappa coefficients and bootstrapped 95\% confidence intervals (Cl's) to infer population validity levels. We interpreted the kappa estimates in acordance with the DSM-5 field trial benchmarks (Kraemer et al. 2012 ).

Table 1: Sensitivity and Specificity of the SCID-II Diagnoses and Scores

\begin{tabular}{lcccccc} 
& $\begin{array}{c}\text { Subjects with the } \\
\text { LEAD Diagnosis } \\
(n=80)\end{array}$ & $\begin{array}{c}\text { Subjects without the } \\
\text { LEAD Diagnosis } \\
(n=40)\end{array}$ & Sensitivity & Specificity \\
SCID-II diagnoses & True (+) & False $(-)$ & False [+] & True [-] & $\%$ & $\%$ \\
\hline Avoidant PD & 14 & 66 & 0 & 40 & 17.5 & 100.0 \\
Dependent PD & 4 & 76 & 0 & 40 & 5.0 & 100.0 \\
Obs-comp. PD & 7 & 73 & 0 & 40 & 8.8 & 100.0 \\
Passive-aggr. PD & 10 & 70 & 2 & 38 & 12.5 & 95.0 \\
Self-defeating PD & 5 & 75 & 1 & 39 & 6.3 & 97.5 \\
Paranoid PD & 21 & 59 & 2 & 38 & 26.3 & 95.0 \\
Schizotypal PD & 4 & 76 & 0 & 40 & 5.0 & 100.0 \\
Schizoid PD & 3 & 77 & 0 & 40 & 3.8 & 100.0 \\
Histrionic PD & 32 & 48 & 3 & 37 & 40.0 & 92.5 \\
Narcissistic PD & 17 & 63 & 1 & 39 & 21.3 & 97.5 \\
Borderline PD & 23 & 57 & 1 & 39 & 28.8 & 97.5 \\
Antisocial PD & 3 & 77 & 1 & 39 & 3.8 & 97.5 \\
Any PD & 72 & 8 & 9 & 31 & 90.0 & 77.5 \\
SCID-II total Scores with applied cut-offs & & & & \\
Cut-off $\geq 19$ & 56 & 24 & 15 & 25 & 70.0 & 62.5 \\
Cut-off $\geq 20$ & 54 & 26 & 13 & 27 & 67.5 & 67.5 \\
\hline
\end{tabular}

\section{Results and Conclusions:}

The diagnostic performance of the SCID-II scores (mean=19.99, $\mathrm{SD}=9.10$, range $=0-42$ ) against LEAD diagnoses was initially investigated with the $\mathrm{ROC}$ analysis which revealed a significant yet unimpressive performance $(\mathrm{AUC}=.74,[95 \% \mathrm{Cl}=.65-.83], \mathrm{p}$ $<.001$ ) and two optimal cut-offs. The sensitivity and specificity values displayed in Table 1 suggested that no SCID-II specific PD diagnosis is conducive by itself to diagnose general PD, yet any $P D$ diagnosis is. To our surprise, optimal cut-offs applied to quantitative SCID-II scores performed worse than any $P D$ diagnosis.

The sample kappas along with their 95\% Cl's are displayed in Figure 1 against red, yellow and green backgrounds indicating poor, questionable/acceptable and satisfactory kappa ranges, respectively. The population kappa estimates excluding unacceptable levels were obtained for none of the specific PD diagnoses, but any PD diagnosis. Also, significantly lower kappa estimates were obtained for the SCID-II scores with the cut-off scores of 19 or 20 , which were being $.31[95 \% \mathrm{Cl}=.14-.47]$ and $.34[95 \% \mathrm{Cl}=.17-.50]$, respectively.

Our data suggest that neither SCID-II specific PD diagnoses nor cut-off applied SCID-II scores, but any PD diagnosis might be instrumental in determining the presence or absence of general PD in the clinical population.

Figure 1: Kappa estimates of the SCID-II PD Diagnoses Against General PD Diagnoses by the LEAD panel.

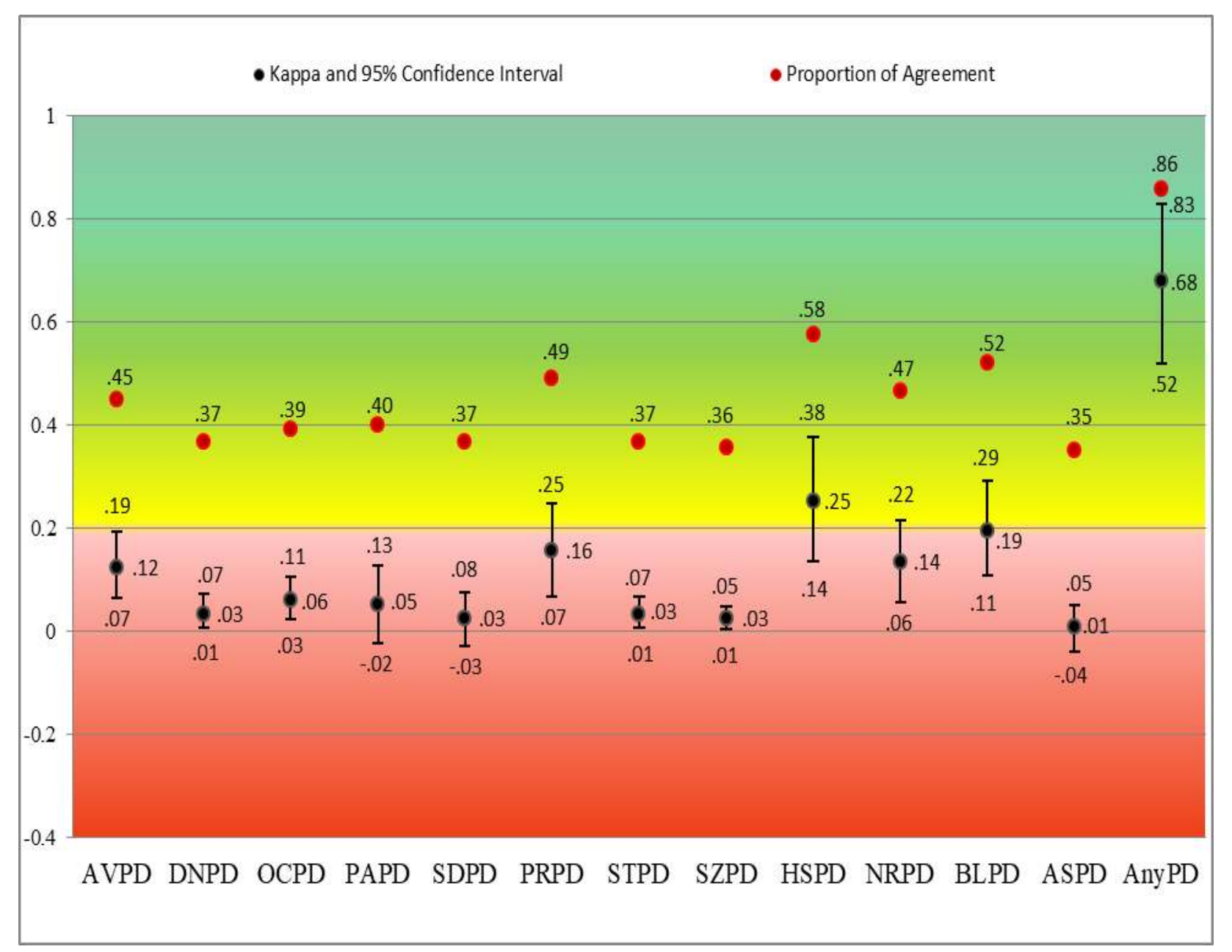

